RESEARCH ARTICLE

\title{
Epidemiology of Dengue Fever among clinically Suspected Febrile Patients at A Tertiary Care Center in Punjab
}

\author{
Shweta Chitkara ${ }^{1}$, Deepinder Chhina ${ }^{1}$, Veenu Gupta ${ }^{1}$, Rajesh Mahajan ${ }^{2}$, Daaman Sharma ${ }^{1}$ \\ ${ }^{1}$ Department of Microbiology, Dayanand Medical College and Hospital, Tagore Nagar, Ludhiana (Punjab), India \\ ${ }^{2}$ Department of Medicine, Dayanand Medical College and Hospital, Tagore Nagar, Ludhiana (Punjab), India
}

\begin{abstract}
Objective: Dengue has emerged as a major public health concern across the globe in terms of mortality and morbidity. The clinical and epidemiological profile of dengue infection changes from time to time. The study was conducted to determine the prevalence of dengue infection, to study the seasonal variation and the clinical profile in these cases.

Methods: A prospective, observational study conducted over a period from April 2015 to March 2016. Patients with acute febrile illness with clinical suspicion of dengue fever admitted in the hospital were included in the study. Serological tests like Dengue IgM and NS1 antigen ELISA were performed to confirm the diagnosis. Serologically confirmed patients of dengue fever were studied for their clinical presentation and lab parameters.

Results: From a total 4732 patients tested for dengue viral infection, $1165(24.6 \%)$ tested positive for dengue. The most affected age group was 26-35 years (25.7\%) with male predominance. Most dengue cases were seen in the months of August to December 2015. Commonly clinical symptoms and signs were myalgia (72.4\%), arthralgia (34.4\%), vomiting (50\%), abdominal pain (38.6\%), hepatomegaly $(27.4 \%)$ and ascites $(10.8 \%)$. Lab parameters revealed leukopenia (43\%) and thrombocytopenia (93.1\%) in most cases. Transaminitis was seen in $84.1 \%$ cases. Common complications were shock (4.8\%) and encephalopathy (4.8\%). Hepatic failure was seen in $3.7 \%$ of patients and renal failure in $1.8 \%$ of patients.
\end{abstract}

Conclusion: Dengue fever has a very non-specific and variable presentation. Knowledge of local prevalence and recent outbreaks can be very helpful in recognizing the clinical entity. J Microbiol Infect Dis 2018; 8(2):43-48

Keywords: Dengue fever, prevalence, seasonality, clinical manifestations, tertiary care hospital

\section{INTRODUCTION}

Dengue fever is one of the most common arbovirus mediated outbreaks, being reported from different parts of the world. Dengue viruses (DV) belong to family Flaviviridae and there are four serotypes of the virus (DV 1-4). It is transmitted mainly by Aedes aegypti mosquito and also by Aedes albopictus [1]. According to WHO estimates approximately 50 million dengue fever (DF) occurs worldwide every year and around 500,000 cases of dengue hemorrhagic fever (DHF) require hospitalization each year with the mortality of $2.5 \%$. India is one of the seven countries in the South-East Asia region regularly reporting incidence of
DF/DHF outbreaks which constantly threatens the health care system [2].

Dengue viruses cause a wide spectrum of clinical disease from a subclinical infection to a mild self-limiting disease, the dengue fever (DF), a severe disease that may be fatal and the dengue hemorrhagic fever/dengue shock syndrome (DHF/DSS) [3]. Differential diagnosis of Dengue fever based on clinical criteria alone is not possible because of overlapping clinical presentations with other tropical fevers and the correct diagnosis is only possible by using pathogen specific diagnostic tests. In resourcelimited settings fever may be treated empirically or self-treated due to lack of access to diagnostic tests. This has a potential for

Correspondence: Dr. Shweta Chitkara, Department of Microbiology, Dayanand Medical College and Hospital, Tagore Nagar, Ludhiana (Punjab)-141001, India

Email: chitkarashweta@gmail.com

Received: 21 February 2017 Accepted: 07 Mai 2018

Copyright (C JMID / Journal of Microbiology and Infectious Diseases 2018, All rights reserved 
increased morbidity and mortality. The major diagnostic methods currently available are viral culture, viral RNA detection by reverse transcriptase PCR (RT-PCR) \& serological tests such as NS1 antigen and IgM capture ELISA. Virus isolation is the gold standard. Although virus isolation provides high sensitivity and specificity, it requires skill and substantial equipment to be carried out and is not the choice for many laboratories [4].

Early laboratory diagnosis of dengue virus infection is routinely done by serological test. The IgM antibody capture ELISA (MAC-ELISA) is based on detecting IgM in serum using human specific $\lg \mathrm{M}$ that is bound to the solid phase. The timing of the sample has great impact on results of IgM ELISA. False negative results are obtained if the sample is collected too early in the infection and false positive results occur because of the cross-reactivity between Flavivirus antibodies [5]. NS1 antigen has gained considerable interest as a new biomarker for early diagnosis of dengue infection [6]. High concentrations of this antigen can be detected in patients with primary and secondary dengue infections up to 9 days after the onset of illness [7].

Knowledge of local prevalence of infections is critical in guiding clinical work up and treatment. The exact burden of dengue in India is not fully known due to limited studies. As effective control and preventive programs for dengue infection are based upon improved surveillance data, the objective of this study was to report the seroprevalence of dengue virus infection at Ludhiana, a major industrial town of North India. We also studied its associated clinical manifestations as well as laboratory findings which would be beneficial in early recognition and proper management of dengue infected patients.

\section{METHODS}

\section{Study population and study design}

This prospective, observational hospital-based study was conducted over a period of one year from April 2015 to March 2016 in the Department of Microbiology, Dayanand Medical College and Hospital, Ludhiana. Blood samples were collected from adult patients $>18$ years of age with febrile illness associated with myalgia, arthralgia, headache, retro-orbital pain, rash, and hemorrhagic manifestations suggestive of dengue fever [WHO criteria] admitted in the hospital.

\section{Sample Collection}

Blood samples were collected by venipuncture taking all aseptic precautions. Venous blood (2-5 $\mathrm{ml}$ ) was collected into a sterile vacutainer without anticoagulant. Serum was separated as soon as possible and refrigerated $\left(2-8^{\circ} \mathrm{C}\right)$ or stored frozen $\left(\leq-20^{\circ} \mathrm{C}\right)$, if not tested within 2 days. Icteric, lipemic, or hemolysed samples were not used. All reagents were brought to room temperature before commencing the assay. The kit expiry was checked before performing the test.

\section{Serology}

The serological tests were done based on the duration of fever and according to the discretion of the treating physician. A total of 4732 samples were screened for dengue virus infection. The Panbio Dengue IgM Capture ELISA (Australia Pty Ltd) was used for qualitative detection of IgM antibodies to dengue antigen in human serum. Dengue NS1 antigen in serum samples was detected using the DENGUE NS1 antigen MICROLISA. These tests were performed and interpreted following the manufacturer's protocol. O.D was measured at $450 \mathrm{~nm}$ using an ELISA reader. Patients who tested positive for either dengue IgM antibody or dengue NS1 antigen by ELISA were considered as confirmed dengue cases [8].

The data was entered in the designated proforma which included the socio-demographic characteristics (age, sex, etc.), presenting clinical symptoms, general physical examination, systemic examination and laboratory investigations were performed. The data obtained was analyzed using descriptive statistics. The Chi-square test was used to find out the $p$ values of the results. $p$ value $<0.05$ was considered significant. Ethical approval was given from the institutional ethical committee.

\section{RESULTS}

A total 4732 blood samples tested for dengue viral infection under study, of which 1165 $(24.6 \%)$ showed positive result for one or more parameters. The most affected age group was 
26-35 years (300 samples, 25.7\%) followed by $36-45$ years (240 samples, $20.6 \%$ ) as shown in Table 1. Males were twice more affected than females. Most dengue cases were seen in the months of August to December 2015 coinciding with the monsoon and post monsoon season. (Figure 1)

The mean duration of fever in dengue cases was $5 \pm 2.38$ days. Patients with dengue commonly presented with symptoms of myalgia (72.4\%), arthralgia (34.4\%), vomiting (50\%) and abdominal pain (38.6\%). Bleeding manifestations were observed in only $10.4 \%$ of patients. Rash was present in $12.1 \%$ of patients. Hepatomegaly was the most common clinical sign observed in $27.4 \%$ of patients. Ascites was seen in $10.8 \%$ of patients. (Table 2 )

Laboratory parameters in dengue cases (Table 3) revealed leukopenia (43\%) and thrombocytopenia (93.1\%) in most cases. Transaminitis was seen in $84.1 \%$ cases. Elevated serum creatinine was seen in $14.6 \%$ of patients. Common complications seen in dengue (Table 4) were shock (4.8\%) and encephalopathy $(4.8 \%)$. Hepatic failure was seen in $3.7 \%$ of patients and renal failure in $1.8 \%$ of patients.

\section{DISCUSSION}

Dengue is an important emerging disease of the tropical and subtropical regions today. Dengue infection has been known to be endemic in many parts of India for over two centuries as a benign and self-limited disease. Epidemics of dengue are increasing in frequency. In our study, the seroprevalence of dengue was $24.6 \%$. In a study by Lall et al, the prevalence of dengue reported over a period of one year was $20.4 \%$ [9]. Garg et al conducted a study in Kanpur in which the prevalence rate of dengue was $19.7 \%$ [10]. Studies from other parts of the country by Ghosh et al, Low et al, and Turbadkar et al have reported similar prevalence of dengue $(17.9 \%, 11.7 \%$ and $13.67 \%$ respectively) [11-13].

Difference in the prevalence rates may be attributed to different geographical areas with different climatic conditions. To identify the seasonal trend of the disease, analysis of the data on monthly basis was done. A gradual increase in cases was noticed from August with a peak in October and decline towards December. The correlation between occurrence of dengue and monsoon season is clearly evident in this study and is further supported by similar findings from other studies [14,15-17]. It may be because this season is very favorable for high breeding of the vector mosquitoes. This seasonal outbreak of disease transmission is very important at local level for effective control measures and that preventive measures against dengue infection should come into full swing during water stagnation periods after the initial bouts of rainfall and at the end of monsoon.

Table 1. Demographic characteristics of dengue cases $(n=1165)$.

\begin{tabular}{lc} 
Age in years & No. of patients (\%) \\
\hline $18-25$ & $203(17.4)$ \\
$26-35$ & $300(25.7)$ \\
$36-45$ & $240(20.6)$ \\
$46-55$ & $216(18.5)$ \\
$56-65$ & $139(12.0)$ \\
$>65$ & $67(5.7)$ \\
Gender & \\
Male & $796(68.3)$ \\
Female & $369(31.7)$ \\
\hline
\end{tabular}

The most affected age group in our study was $26-35$ years $(300,25.7 \%)$ followed by $36-45$ years $(240,20.6 \%)$. These age groups are the active working population who are most likely to be exposed to tropical infections. Also males were twice more affected than females. This may be due to their easy exposure to mosquitoes and mites because of their outdoor activities. Similar findings have been reported in studies by Chrispal et al, Ittyachen et al and others, with most of the patients being in the productive phase of life (18-45 years) and a male preponderance $[14,18.19]$.

Common clinical symptoms of dengue in our study were myalgia (72.4\%), arthralgia (34.4\%), vomiting $(50 \%)$, abdominal pain $(38.6 \%)$ and decreased urine output $(7 \%)$. Mean duration of fever was 5 days. Rash was present in $12.1 \%$ patients. Headache was seen less frequently (15.7\%). Hepatomegaly (27.4\%) and ascites $(10.8 \%)$ were common clinical signs observed. Ghosh et al observed that the typical clinical features of dengue included chills and rigors $(80 \%)$, myalgia (70\%), vomiting $(60 \%)$, headache (50\%) and rash (25\%) [20]. Similar clinical picture was reported by Shah et al [21] 
Table 2: Spectrum of clinical symptoms and signs in Dengue $(n=1,165)$

\begin{tabular}{|c|c|c|}
\hline Clinical symptoms & No. of patients (\%) & p value \\
\hline Myalgia & $844(72.4)$ & $<0.0001$ \\
\hline Arthralgia & $401(34.4)$ & $<0.0001$ \\
\hline Headache & $184(15.7)$ & $<0.0001$ \\
\hline Abdominal pain & $450(38.6)$ & 0.0004 \\
\hline Vomiting & $582(50)$ & $<0.0001$ \\
\hline Jaundice & $88(7.5)$ & $<0.0001$ \\
\hline Bleeding manifestations & $122(10.4)$ & 0.84 \\
\hline - $\quad$ Petechiae & $21(1.8)$ & \\
\hline Malena & 38 (3.2) & \\
\hline Hematemesis & $38(3.2)$ & \\
\hline Hemoptysis & $8(0.7)$ & \\
\hline Gum bleed & $1(0.08)$ & \\
\hline - $\quad$ Epistaxis & $7(0.6)$ & \\
\hline Decreased urine output & $81(7)$ & 0.0003 \\
\hline Cough & $62(5.3)$ & 0.51 \\
\hline Breathlessness & $48(4.1)$ & 0.42 \\
\hline Altered sensorium & $59(5)$ & 0.02 \\
\hline \multicolumn{3}{|l|}{ Clinical signs } \\
\hline Rash & $142(12.1)$ & $<0.0001$ \\
\hline Abdominal tenderness & $148(12.7)$ & 0.04 \\
\hline Abdominal distension & $50(4.2)$ & 0.02 \\
\hline Hepatomegaly & $320(27.4)$ & $<0.0001$ \\
\hline Splenomegaly & $14(1.2)$ & 0.84 \\
\hline Ascites & $126(10.8)$ & $<0.0001$ \\
\hline Chest crepitation & 31 & $<0.0001$ \\
\hline
\end{tabular}

Table 3: Lab parameters in Dengue $(n=1,165)$.

\begin{tabular}{|c|c|c|c|}
\hline \multicolumn{2}{|l|}{ Lab parameters } & No. of patients (\%) & p value \\
\hline \multirow[t]{2}{*}{ TLC (cells/ $\mu \mathrm{L})$} & Leukopenia $(<4000)$ & $502(43)$ & $<0.0001$ \\
\hline & Leukocytosis (>11000) & $81(7)$ & $<0.0001$ \\
\hline \multirow[t]{2}{*}{ Platelets (cells/ $\mu \mathrm{L})$} & Thrombocytopenia, $<100 \times 10^{3}$ & $1085(93.1)$ & $<0.0001$ \\
\hline & $\geq 100 \times 10^{3}$ & $78(6.6)$ & $<0.0001$ \\
\hline \multicolumn{2}{|c|}{ SGOT >50 and SGPT >50 (U/L) } & $980(84.1)$ & $<0.0001$ \\
\hline \multicolumn{2}{|c|}{ Total bilirubin >1.2 (mg/dl) } & $234(20)$ & $<0.0001$ \\
\hline \multicolumn{2}{|c|}{ Urea $>50(\mathrm{mg} / \mathrm{dL})$} & $139(12)$ & $<0.0001$ \\
\hline \multicolumn{2}{|c|}{ Creatinine $>1.2(\mathrm{mg} / \mathrm{dL})$} & $171(14.6)$ & $<0.0001$ \\
\hline
\end{tabular}

Table 4: Complications in Dengue $(n=1,165)$.

\begin{tabular}{lcl}
\hline Complication & No. of patients (\%) & Ialue \\
\hline Shock & $57(4.8)$ & 1.58 \\
Encephalopathy & $56(4.8)$ & 1.26 \\
Hepatic failure & $44(3.7)$ & .0001 \\
Renal failure & $22(1.8)$ & .0001 \\
Pleural effusion & $31(2.6)$ & .0001 \\
ARDS & $5(0.4)$ & 1.07 \\
Sepsis & $2(0.1)$ & .002 \\
\hline $\mathrm{n}=$ serologically confirmed dengue cases, $\mathrm{p}$ value $<0.05$ is \\
significant
\end{tabular}

Seropositivity of dengue

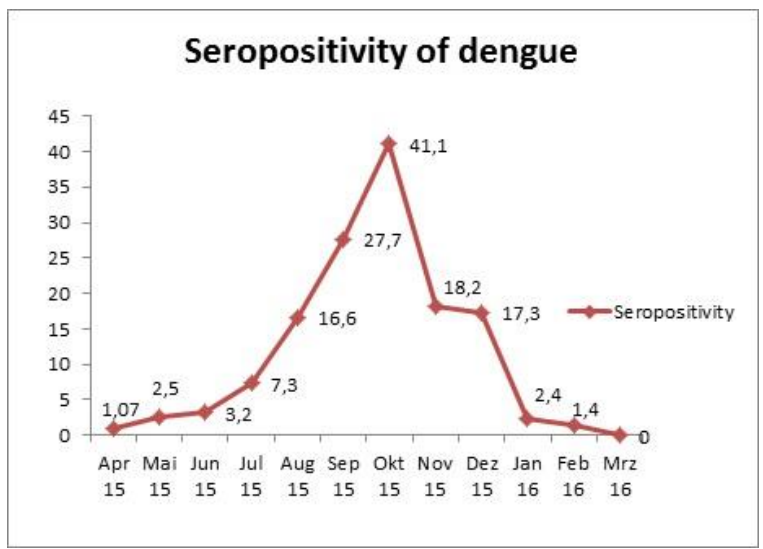

Figure 1: Month wise positivity of dengue $(n=1165)$.

Bleeding manifestations were observed in only $10.4 \%$ of patients in our study, which was statistically not significant. The most common 
form was gastrointestinal bleed like melena and hematemesis. Khan et al also reported only 5\% patients with bleeding while $40 \%$ patients had thrombocytopenia [22]. This is in contrast to study by Gupta et al which reported symptoms of melena $(50 \%)$ and hematemesis $(38 \%)$ during the 2006 outbreak of dengue in North India [23].

In our study lab parameters in dengue patients revealed mostly leukopenia (43\%) and thrombocytopenia $(93.1 \%)$. Transaminitis was also common in $84.1 \%$ cases. These findings were in congruence with findings of other studies by Tejaswi et al [24] and Chatterjee et al [25] who revealed gross leukopenia, thrombocytopenia and elevated transaminases as significant lab derangements in dengue cases. Shock (4.8\%) and encephalopathy $(4.8 \%)$ were common complications observed in patients of dengue in our study. Hepatic failure was observed in $3.7 \%$ and renal failure in $1.8 \%$ of patients. Singh et al reported complications like shock in $4.2 \%$ and ARDS in $2.1 \%$ of patients [26]. Complications seen in the study by Kashinkunti et al were hepatic dysfunction $(34 \%)$, renal failure $(26 \%)$, multi organ failure $(18 \%)$, encephalopathy (13\%) and ARDS in (12\%) [18].

The present study highlights the importance of recognizing the varied manifestations of dengue fever and its complications to the clinicians for its timely diagnosis and management.

As conclusion, Dengue fever is an emerging public health problem in tropical countries like India and it can present with varied acute clinical manifestations. A high index of suspicion is required to detect and timely manage the DF. The results of this study describe the acute clinical manifestations and role of laboratory investigations in DF patients. Hence, continuous seroepidemiological surveillance and timely interventions are needed to identify the cases so that its complications, outbreak, and mortality can be minimized.

\section{ACKNOWLEDGMENTS}

Conflict of interest: The authors declare no personal or financial conflict of interest.

Financial Support: The authors declared no financial support.

\section{REFERENCES}

1. Centers for Disease Control and Prevention [Internet]. Centers for Disease Control and Prevention. Centers for Disease Control and Prevention; 2016 [cited 2016 Nov 9]. Available from: http://www.cdc.gov/dengue/entomologyecology/index. html

2. World Health Organization. Dengue and Dengue Hemorrhagic fever [Internet]. WHO; March 2014 [cited 2016 Nov 9]. Available from http:// www.who.int/mediacentre/factsheets/fs117/en/

3. Guzmán MG, Kouri G. Dengue: an update. The Lancet infectious diseases. 2002; 2(1):33-42.

4. Shamala DS. Laboratory diagnosis of Dengue: A review. The international medical Journal Malaysia. 2015; 14:17-28.

5. Peeling RW, Artsob H, Pelegrino JL, et al. Evaluation of diagnostic tests: dengue. Natur Rev Microbiol 2010; 8:30-37.

6. Subedi D, Taylor-Robinson AW. Laboratory Diagnosis of Dengue Infection: Current Techniques and Future Strategies. Open J Clin Diagn 2014; 4:63-70.

7. Dussart P, Labeau B, Lagathu G, et al. Evaluation of an Enzyme Immunoassay for Detection of Dengue Virus NS1 Antigen in Human Serum. Clinical and Vaccine Immunology. 2006;13(11):1185-9.

8. National Guidelines for Clinical Management of Dengue Fever [Internet]. [cited 2016Nov17]. Available from: http://nvbdcp.gov.in/doc/dengue-nationalguidelines-2014.pdf

9. Lall H, Jais M, Gupta P. Sero-Prevalence of Dengue in Tertiary Care Hospital in Delhi. Int J Curr Microbiol App Sci 2016; 5(6):439-445.

10. Garg A, Garg J, Rao YK, Upadhyay GC, Sakhuja S. Prevalence of dengue among clinically suspected febrile episodes at a teaching hospital in North India. Journal of Infectious Diseases and Immunity. 2011; 3(5):85-89.

11. Ghosh G, Urhekar AD, Kosta S. A clinicomicrobiological study of dengue fever cases in a tertiary care center of navi Mumbai. International Journal of Bioassays. 2013; 2(11):1462-1467.

12. Low JG, Ong A, Tan LK, et al. The early clinical features of dengue in adults: challenges for early clinical diagnosis. PLoS Negl Trop Dis 2011; 5(5):e1191.

13. Turbadkar D, Ramchandran A, Mathur M, Gaikwad S. Laboratory and clinical profile of dengue: A study from Mumbai. Annals of Tropical Medicine and Public Health. 2012; 5(1):20.

14. Chrispal A, Boorugu $H$, Gopinath KG, et al. Acute undifferentiated febrile illness in adult hospitalized patients: the disease spectrum and diagnostic predictors - an experience from a tertiary care 
hospital in South India. Trop Doctor 2010; 40(4):230234.

15. Jena B, Prasad M, Murthy S, Ramanarao G. Demand pattern of Medical Emergency Services for Infectious Diseases in Andhra Pradesh-A Geo-spatial Temporal Analysis of Fever cases. Transforming Emergency Management. Indian Emergency Journal. 2010; 1(5):821

16. Murdoch DR, Woods CW, Zimmerman MD, et al. The aetiology of febrile illness in adults presenting to Patan Hospital in Kathmandu, Nepal. Am J Trop Med Hyg 2004; 70(6):670-675.

17. Zimmerman MD, Murdoch DR, Rozmajzl PJ, et al. Murine typhus and febrile illness, Nepal. Emerg Infect Dis 2008; 14(10):1656-9.

18. Kashinkunti MD, Gundikeri SK, Dhananjaya M. Acute undifferentiated febrile illness- clinical spectrum and outcome from a tertiary care teaching hospital of north Karnataka. Int J Biol Med Res 2013; 4(2):3399-4002.

19. Ittyachen AM, Ramachandran R. Study of acute febrile illness: a 10-year descriptive study and a proposed algorithm from a tertiary care referral hospital in rural Kerala in Southern India. Trop Doct 2015; 45(2):114-117.

20. Ghosh G, Urhekar AD, Kosta S. A clinicomicrobiological study of dengue fever cases in a tertiary care center of navi Mumbai. Int $\mathrm{J}$ Bioassay 2013; 2(11):1462-1467.

21. Low JG, Ong A, Tan LK, et al. The early clinical features of dengue in adults: challenges for early clinical diagnosis. PLoS Negl Trop Dis 2011; 5(5):e1191.

22. Turbadkar D, Ramchandran A, Mathur M, Gaikwad S. Laboratory and clinical profile of dengue: A study from Mumbai. Annals of Tropical Medicine and Public Health. 2012; 5(1):20.

23. Gupta $P$, Trikha A. The north Indian dengue outbreak 2006: a retrospective analysis of intensive care unit admissions in a tertiary care hospital. Transactions of the Royal Society of Tropical Medicine and Hygiene. 2008; 102(2):143-147.

24. Tejaswi CN, Patil SS, Shekharappa KR. Study of clinical manifestations of dengue cases in a tertiary care hospital, Bangalore, Karnataka. International Journal of Medical Science and Public Health 2016; 5(12).

25. Chatterjee N, Mukhopadhyay M, Ghosh S, Mondol M, Das C, Patar K. An observational study of dengue fever in a tertiary care hospital of Eastern India. JAPI 2014; 62:224-227.

26. Singh R, Singh SP, Ahmad N. A study of clinical and laboratory profile of dengue fever in a tertiary care centre of Uttarakhand, India. Int J Resear Med Sci 2014;2 (1):160-163. 